\title{
Inflammasome Priming Mediated via Toll-Like Receptors 2 and 4, Induces Th1-Like Regulatory T Cells in De Novo Autoimmune Hepatitis
}

\author{
Adam S. Arterbery', Jie Yao', Andrew Ling ${ }^{1}$, Yaron Avitzur ${ }^{2}$, Mercedes Martinez ${ }^{3}$, \\ Steven Lobritto ${ }^{3}$, Yanhong Deng ${ }^{4}$, Gan Geliang ${ }^{4}$, Sameet Mehta ${ }^{5}$, Guilin Wang ${ }^{5}$, \\ James Knight ${ }^{5}$ and Udeme D. Ekong ${ }^{1 *}$
}

${ }^{1}$ Pediatric Gastroenterology and Hepatology, Yale University, New Haven, CT, United States, ${ }^{2}$ Gastroenterology, Hepatology, and Nutrition, Hospital for Sick Children, Toronto, ON, Canada, ${ }^{3}$ Pediatric Gastroenterology, Hepatology, and Nutrition, Columbia University, New York, NY, United States, ${ }^{4}$ Yale Center for Analytical Sciences, New Haven, CT, United States,

${ }^{5}$ Yale Center for Genome Analysis, Yale School of Medicine, New Haven, CT, United States

OPEN ACCESS

Edited by:

Heiko Mühl,

Goethe-Universität Frankfurt am

Main, Germany

Reviewed by:

Maria Serena Longhi,

Harvard Medical School,

United States

Pascal Lapierre,

University of Montreal Hospital

Centre (CRCHUM), Canada

*Correspondence:

Udeme D. Ekong

udeme.ekong@yale.edu

Specialty section: This article was submitted

to Inflammation,

a section of the journal

Frontiers in Immunology

Received: 12 April 2018 Accepted: 28 June 2018

Published: 19 July 2018

Citation:

Arterbery AS, Yao J, Ling A, Avitzur Y,

Martinez M, Lobritto S, Deng $Y$,

Geliang G, Mehta S, Wang G,

Knight $J$ and Ekong UD (2018) Inflammasome Priming Mediated via

Toll-Like Receptors 2 and 4, Induces

Th1-Like Regulatory T Cells in

De Novo Autoimmune Hepatitis.

Front. Immunol. 9:1612.

doi: 10.3389/fimmu.2018.01612
De novo autoimmune hepatitis (DAlH) is an important cause of late allograft dysfunction following liver transplantation, but its cause and underlying pathogenesis remains unclear. We sought to identify specific innate and adaptive immune mechanisms driving the pro-inflammatory cytokine secreting regulatory $T$ cell (Treg) phenotype in DAlH and determine if modulation of these pathways could resolve the inflammatory milieu observed in the livers of patients with DAlH. Here, we demonstrate toll-like receptors (TLRs) 2- and 4-mediated inflammasome activation in $\mathrm{CD}_{14^{++}}$monocytes, a finding that is key to maintaining dysfunctional Tregs in patients with DAlH. Furthermore, silencing of TLR 2 and 4 in $\mathrm{CD}_{14}{ }^{++}$monocytes prevented activation of the inflammasome and significantly decreased IFN- $\gamma$ production by FOXP3 ${ }^{+}$Tregs. We also observed significantly increase in expression of tumor necrosis factor $\alpha$-induced protein 3 (TNFAIP3), a negative regulator of the NLRP3 Inflammasome, in monocytes/macrophages of liver transplant subjects who have normal allograft function and do not have DAlH. TNFAIP3 expression was virtually absent in monocytes/macrophages of patients with $\mathrm{DAlH}$. Our findings suggest that autoimmunity in DAlH is promoted by $\mathrm{CD} 14^{++}$monocytes predominantly through activation of inflammatory signaling pathways.

Keywords: innate immunity, toll-like receptors, inflammasome, Th1 regulatory $\mathrm{T}$ cells, liver transplantation, $\mathrm{CD}_{14}{ }^{++}$monocytes, autoimmune hepatitis, tumor necrosis factor $\alpha$-induced protein 3

\section{INTRODUCTION}

De novo autoimmune hepatitis (DAIH) is an important cause of late allograft dysfunction after liver transplantation, but its cause and underlying pathogenesis remains unclear. We have previously reported that $\mathrm{CD}_{14}{ }^{++}$monocyte-derived IL-12, directs regulatory T cells (Tregs) to differentiate into dysfunctional, IFN- $\gamma$ secreting, Th1-like effector Tregs (1). Furthermore, we observed that these monocytes as well as $\mathrm{CD}^{+} 8^{+}$macrophages in the liver constitutively produce IL-1 $\beta$ (1). To better understand the mechanisms driving $\mathrm{CD} 14^{++}$monocyte activation and Treg differentiation, we sought to identify specific innate and adaptive immune mechanisms that drive a pro-inflammatory, cytokine secreting, effector Treg phenotype. In addition, we examined whether modulation of these pathways 
could resolve the inflammatory milieu observed in the livers of patients with DAIH. Given that IL- $1 \beta$ is a signature downstream cytokine of inflammasome activation $(2,3)$, we hypothesized that activation of the innate immune system is the driver for the adaptive system in DAIH and that the chronic inflammation in DAIH is augmented by inflammasomes. Our results show that priming of the inflammasome mediated via toll-like receptors (TLRs) 2 and 4 is increased in DAIH and autoimmune hepatitis (AIH) and is key to maintaining dysfunctional Tregs. Our findings provide further insights into the balance and communication between innate and adaptive immune responses in DAIH that may lead to potential therapeutic options.

\section{MATERIALS AND METHODS}

\section{Study Design}

This was a cross sectional study involving pediatric liver transplant recipients with a diagnosis of DAIH $(n=11)$, pediatric liver transplant recipients who had never been diagnosed with DAIH and had normal allograft function at the time of enrollment and blood draw. These subjects acted as the liver transplant control group (LTC) $(n=42)$, and healthy children who had no underlying immune-mediated disorders and were not on any immunomodulatory agents. These subjects acted as the healthy control group (HC) $(n=39)$. Finally, non-transplanted children with a diagnosis of AIH were also included $(n=9)$. The transplanted subjects were enrolled as they attended their routine post-transplant clinics, the non-transplanted children with $\mathrm{AIH}$ were enrolled as they attended their routine pediatric hepatology clinics, and the healthy non-transplanted subjects were enrolled using flyers posted in New Haven as well as from the help us discover database, a database of healthy children registered as research controls maintained by the Yale Center for Clinical Investigation. The definition of DAIH was as previously described (4): (a liver transplant recipient without a history of autoimmune liver disease presenting with unknown etiology of late graft dysfunction. Late graft dysfunction characterized by elevated aminotransferases, and graft dysfunction not due to any of the following causes: acute and chronic rejection, hepatitis B and C infection, Epstein Barr virus and Cytomegalovirus infections, vascular problems, biliary complication, drug toxicity, sepsis, recurrence of primary disease, or post-transplant lymphoproliferative disease; elevated serum immunoglobulin $\mathrm{G}$, positive autoantibody titers: ANA, ASMA, anti-LKM; characteristic biopsy findings of dense lymphocytic portal tract infiltrate with plasma cells, and interface hepatitis) (4). The definition of AIH was as previously described $(5,6)$. Inclusion/exclusion criteria were established prospectively.

The inclusion criteria for the liver transplant control group were age $\leq 20$ years, serum aminotransferases and gamma glutamyl transpeptidase $\leq \mathrm{ULN}$ at blood draw, no past diagnosis of DAIH, no biliary or vascular complication at time of blood draw, no past or present history of chronic rejection, no recent acute rejection ( $>1$ year). The exclusion criteria included age $\geq 21$ years, biliary/ vascular complication at time of blood draw, serum aminotransferases and gamma glutamyl transpeptidase $>$ ULN at blood draw, recent acute rejection ( $\leq 1$ year), and history of chronic rejection. We enrolled patients aged 0 through 20 years from January 2015 to December 2017. The rule for stopping patient enrollment and sample collection was determined by the dates as stated above. At enrollment, $30 \mathrm{~mL}$ of peripheral blood was obtained from each participant, and every 3 months over the duration of the study. If the study participant underwent a clinically indicated liver biopsy, liver tissue was obtained for isolation of intrahepatic lymphocytes. Each experiment described was replicated twice with substantiation of the results. Informed consent was obtained from parents or guardians and assent was obtained as necessary per institutional review board guidelines. The study protocol was reviewed and approved by the Institutional Review Board of Yale University.

\section{Monocyte Stimulation Experiments}

$\mathrm{CD}_{1} 4^{++}$monocytes were isolated, stimulated, stained, and subjected to FACS analysis for IL- $1 \beta$ as previously described (1).

\section{Western Blotting}

$\mathrm{CD}_{14}{ }^{++}$monocytes were isolated and stimulated as previously reported (1), and protein was harvested using MPER solution with PhosSTOP according to the manufacturer's instructions. Protein was harvested in M-PER protein extraction reagent supplemented with PhosSTOP phosphatase inhibitor tablets according to the manufacturer's instructions. Protein concentrations were measured using the Bradford method and equal amounts of protein were analyzed by western blotting. For CD1 $4^{++}$monocytes, the following inflammasome-associated antibodies were used: NLRP3, ASC, (Pro) IL-1 $\beta$, and (Pro) Caspase 1. All bands were subjected to densitometry using ImageJ, and referenced against GAPDH.

\section{Quantification of mRNA Expression Levels by RT-PCR}

qRT-PCR was carried out as previously reported (1). Briefly, RNA was isolated using Qiagen RNeasy Micro Kit (Qiagen), following manufacturer's guidelines and converted to cDNA using qScript cDNA Supermix according to the manufacturer's instructions. For quantification of damage-associated molecular patterns (DAMPs), DNA was harvested from $200 \mu \mathrm{L}$ of patient sera using QIAamp DNA Blood Mini Kit according to the manufacturer's instructions. The following were targeted: DAMPs-HMGB1, MT-ATP6, ACTB, Fibrinogen, HSP60, HSP70, and HSP90. In addition, pro- $I L-1 \beta$ was quantified in $\mathrm{CD} 14^{++}$monocytes stimulated with lipopolysaccharide (LPS) $(1 \mathrm{mg} / \mathrm{mL})$ for $24 \mathrm{~h}$. Probes and cDNA were mixed with SsoAdvanced Universal Probes Supermix and the reactions were set up following manufacturer's guidelines and run on a 7500 Fast Real-Time PCR System (Applied Biosystems). Values are represented as the difference in cycle threshold values normalized to reference gene $(\beta 2 \mathrm{M}$ or $18 \mathrm{~s})$ for each sample as per the following formula: relative RNA expression $=\left(2^{-\mathrm{dCt}}\right) \times 1,000$.

\section{Luminex-ELISA}

Frozen plasma samples were thawed completely and centrifuged for $5 \mathrm{~min}$ prior to addition to the assay. Three Milliplex Map kits (EMD Millipore, Bilerica, MA, USA) were used to measure 
mitochondrial proteins in one assay (Complexes I-IV, Human Oxidative Phosphorylation Magnetic Bead Panel); heat shock proteins in another (Heat Shock Protein Magnetic Bead 5-Plex Kit); Fibrinogen protein in the third (Human Cardiovascular Disease Magnetic Bead Panel 3-Fibrinogen 1 Plex); and S100A8 and S100A12 in the fourth and fifth kit, respectively. Samples were incubated overnight at $4^{\circ} \mathrm{C}$ with a mixture of magnetic beads coupled to an antibody specific for one of the analytes. After washing the beads in the 96-well plates using a hand held magnet, the beads were incubated for $1 \mathrm{~h}$ at room temperature with biotinylated detection antibodies, washed, and then incubated for $30 \mathrm{~min}$ at room temperature with streptavidin-phycoerythrin. Plates were read in a Luminex 200 Analyzer (Luminex, Austin, TX, USA) controlled by xPONENT software. Values for each analyte were determined using Analyst software (EMD Millipore) from a standard curve of log dose vs. median fluorescent intensity using a five parameter logistic fit. All standards and samples were run in duplicate. The standard curve was used to calculate the concentration of QC controls supplied with the kits and found to be in the expected range.

\section{TLR Reporter Cell Lines and Sera Treatment (Inhibition)}

Stimulation by proteins in patient sera: HEK cells that stably co-express a human TLR2, TLR4, or TLR9 gene and an NF- $\kappa \mathrm{B}-$ inducible secreted embryonic alkaline phosphatase (SEAP) reporter gene were used to determine activation of TLRs from patient sera. Expression of SEAP under control of NF-кB/AP-1 promoters is inducible by TLR activation, and extracellular SEAP in the supernatant is proportional to NF- $\kappa B$ induction. Secreted SEAP levels quantified in the supernatant on the basis of optical density (colorimetric intensity) at $650 \mathrm{~nm}$. Positive controls were as follows: TLR2-FSL1 $(100 \mu \mathrm{g} / \mathrm{mL})$, TLR4-LPS $(100 \mathrm{ng} / \mathrm{mL})$, and TLR9-OD2006 $(100 \mu \mathrm{g} / \mathrm{mL})$. Following the culture of TLR reporter cell lines according to the manufacturer's instructions, $50 \mu \mathrm{L}$ of sera (diluted 1:1 with PBS) was added and cultured for $24 \mathrm{~h}$. The following day, $50 \mu \mathrm{L}$ of supernatant was withdrawn from each culture, added to $200 \mu \mathrm{L}$ Qunati-Blue, and incubated according to the manufacturer's instructions. Colorimetric changes were observed and secreted SEAP levels quantified in the supernatant on the basis of optical density (colorimetric intensity) at $650 \mathrm{~nm}$. Inhibition of proteins in patient sera: TLR reporter lines were cultured with patient sera as above. Protein inhibitors for Fibrinogen, HSPs (90), and Mitochondrial Complexes were applied to the cell cultures for $48 \mathrm{~h}$ at the following concentrations: $1 \mathrm{~mm}$ 17AAG (HSPs); $10 \mu \mathrm{M}$ Fibrinogen Inhibitor (Fibrinogen); $25 \mu \mathrm{M}$ Rotenone (Complex I); $10 \mathrm{mM}$ TTFA (Complex II); $20 \mu \mathrm{M}$ Antimycin A (Complex III). Colorimetric changes were observed and secreted SEAP levels quantified in the supernatant on the basis of optical density (colorimetric intensity) at $650 \mathrm{~nm}$.

\section{Inhibition of TLRs in CD14 ${ }^{++}$Monocytes Using shRNA}

Lentiviral particles expressing shRNAs were obtained from Origene (Rockville, MD, USA) for TLR2, TLR4, and TLR9. CD14 ${ }^{++}$ monocytes were subject to the recommended manufacturer's protocol for transduction of non-adherent primary cells. In brief, $\mathrm{CD}_{14}{ }^{++}$monocytes were obtained by positive selection (18058; STEMCELL) from peripheral blood mononuclear cells (PBMCs) following manufacturer's guidelines. For transduction, $5 \times 10^{4}$ human $\mathrm{CD} 14^{++}$monocytes were transduced with viral particles containing a vector expressing TLR-specific shRNA or as controls a vector expressing an unspecific shRNA. Transduction was mediated at a multiplicity of infection of 5 by centrifugation at $2,250 \mathrm{rpm}$ for $30 \mathrm{~min}$ at room temperature in the presence of $3 \mu \mathrm{g} / \mathrm{mL}$ polybrene (Millipore, Billerica, MA, USA). Cells were then cultured in Xvivo15 media with LPS $(1 \mathrm{mg} / \mathrm{mL})$ for $24 \mathrm{~h}$ at $37^{\circ} \mathrm{C}$ with $5 \% \mathrm{CO}_{2}$. The following day, cells were gently resuspended and one-third the volume was harvested for RNA, converted to cDNA, and subjected to qRT-PCR as described above; and analyzed for the expression of TR2, TLR4, and TLR9, respectively. Following the confirmation of knockdown by qRT-PCR, 10,000 transduced $\mathrm{CD} 14^{++}$monocytes were then co-cultured with $50,000 \mathrm{CD} 4{ }^{+} \mathrm{CD} 25^{\mathrm{hi}} \mathrm{CD} 127^{-}$Tregs for 5 days as previously described (1). Cells were then subjected to intracellular staining of cytokine, Tregs were analyzed for expression of FOXP3 and IFN- $\gamma$ expression, and $\mathrm{CD} 14^{++}$monocytes were analyzed for IL-6, IL-12, and IL-1 $\beta$ expression using flow cytometry as previously described (1); or cells were harvested for RNA for analysis of TLR gene expression by qPCR and confirmation of transient knockdown. In addition, $\mathrm{CD} 14^{++}$monocytes were analyzed by western blot for inflammasome-associated proteins as described above.

\section{Single-Cell Sequencing}

$\mathrm{CD} 14^{++}$monocytes from $\mathrm{PBMCs}$ and $\mathrm{CD}^{+} 8^{+}$macrophages from human liver were FACS sorted for use in single-cell sequencing. Briefly, sorted cells were counted and assessed for viability with Trypan Blue using a Countess II automated counter (Invitrogen), and adjusted at a concentration of $800-1,000$ cells/ $\mu \mathrm{L}$. Final cell viability estimates ranged between 85 and 95\%. To generate single-cell gel beads in emulsion (GEMs) and sequencing libraries, single-cell suspensions were loaded onto 10X Genomics Single Cell A Chips (10x Genomics, PN-120236) along with the reverse transcription master mix as per the manufacturer's protocol for the Chromium Single Cell 3' Reagent kits V2 (10X Genomics; PN-120237). Libraries were sequenced on an Illumina HiSeq2500 using rapid mode as follows: 26 bp (Read 1); 8 bp (i7 Sample Index); 0 bp (i5 Index); and 98 bp (Reads 2). The Cell Ranger Single Cell Software Suite 2.1.0 by 10X Genomics (http://10xgenomics.com/) was used to process sequenced data into transcript count tables. Downstream processing and visualization was done using $\mathrm{R}$ and Seurat.

\section{Hepatocyte and CD14 ${ }^{++}$Monocyte Co-Culture}

In brief, $\mathrm{CD} 14^{++}$monocytes were obtained by positive selection from PBMCs following manufacturer's guidelines from subjects with DAIH, liver transplanted control (LTC) subjects and healthy, non-transplanted subjects. $\mathrm{CD} 14^{++}$monocytes were then cocultured with normal human hepatocytes (obtained through the Liver Tissue Cell Distribution System, University of Pittsburgh, 
PA, USA) in a 1:4 ratio (monocyte:hepatocyte), for 24,72 , and $96 \mathrm{~h}$ at $37^{\circ} \mathrm{C}$ with $5 \% \mathrm{CO}_{2}$; Cultured cells were then subjected to confocal microscopy for detection of anti-active caspase-3, and anti-CD14 as follows: cultured cells rinsed with PBS and fixed with methanol for $2 \mathrm{~h}$ on ice, subsequently incubated with the primary antibody overnight at $4^{\circ} \mathrm{C}$, and secondary antibody over $2 \mathrm{~h}$ at room temperature. Primary and secondary antibodies were anti-human albumin antibody with Alexa Fluor 488-labeled IgG, anti-caspase-3 antibody with Alexa Fluor 555-labeled IgG, and anti-CD14 antibody with Alexa Fluor 647-labeled IgG, respectively. Imaging performed using a Leica YSCC SP5 confocal system at $100 \times$ objective. Culture supernatant was obtained for measurement of alanine aminotransferase. Measurement of alanine aminotransferase was performed using the Alfa Wassermann automated chemistry analyzer (Alera model).

\section{RESULTS}

\section{Demographics}

Table 1 highlights the demographics of all enrolled subjects. For the transplanted subjects, six were on Sirolimus (two subjects with DAIH and four LTC subjects), and four on Cyclosporine (two subjects each in the DAIH and LTC group, respectively). The remaining transplanted subjects were all on Tacrolimus. Importantly there was no significant difference in levels of antirejection drugs and pre-transplant diagnosis between the two liver transplanted subject groups.

\section{Inflammasome Activation Occurs in CD14 ${ }^{++}$Monocytes From Patients With DAIH}

Given our published observation of constitutive IL-1 $\beta$ production in $\mathrm{CD}_{14}^{++}$monocytes of patients with DAIH (1), we investigated for inflammasome activation in $\mathrm{CD}^{+4^{++}}$monocytes. CD $14^{++}$monocytes from subjects with DAIH produced significantly more IL-1 $\beta$ and expressed significantly more pro$I L-1 \beta$ compared to $\mathrm{CD} 14^{++}$monocytes from liver transplanted patients with normal allograft function who did not have DAIH (LTC) and $\mathrm{CD}_{14}{ }^{++}$monocytes from healthy, non-transplanted children (HC) (Figures 1A,B) (gating strategy Figure S1A in Supplementary Material).

We also observed significantly increased expression of inflammasome-associated effector proteins from $\mathrm{CD} 14^{++}$monocytes of patients with DAIH compared to expression in $\mathrm{CD} 14^{++}$ monocytes from LTC and HC (Figures 1C,D). Together these results demonstrate increased caspase-1 cleavage, and expression of the downstream signature cytokine of inflammasome activation, IL- $1 \beta$ in $\mathrm{CD} 14^{++}$monocytes of patients with DAIH. Of note, we also observed similar caspase- 1 cleavage with IL- $1 \beta$ secretion from $\mathrm{CD} 14^{++}$monocytes of non-transplanted subjects with $\mathrm{AIH}$ compared to $\mathrm{CD} 14^{++}$monocytes of healthy, non-transplanted children (Figure S1B in Supplementary Material). Thus, pathologic inflammasome activation in $\mathrm{CD}_{1} 4^{++}$monocytes appears to be a common feature in at least two forms of autoimmune liver disease.

\section{Inflammasome Activation Mediated by TLRs 2/4 and 9 in DAIH}

To further understand the mechanism underlying inflammasome activation in DAIH, we looked for the presence of DAMPs in sera and found sera of patients with DAIH had a significant abundance of DAMPs known to activate TLRs 2/4 and 9 (3) (Figures 2A,B) compared to sera of LTC subjects, and compared to sera of healthy, non-transplanted children (HC). Similarly, in non-transplanted children with AIH, DAMPs that activate TLR's, 2, 4, and 9 were significantly increased in

TABLE 1 | Demographics.

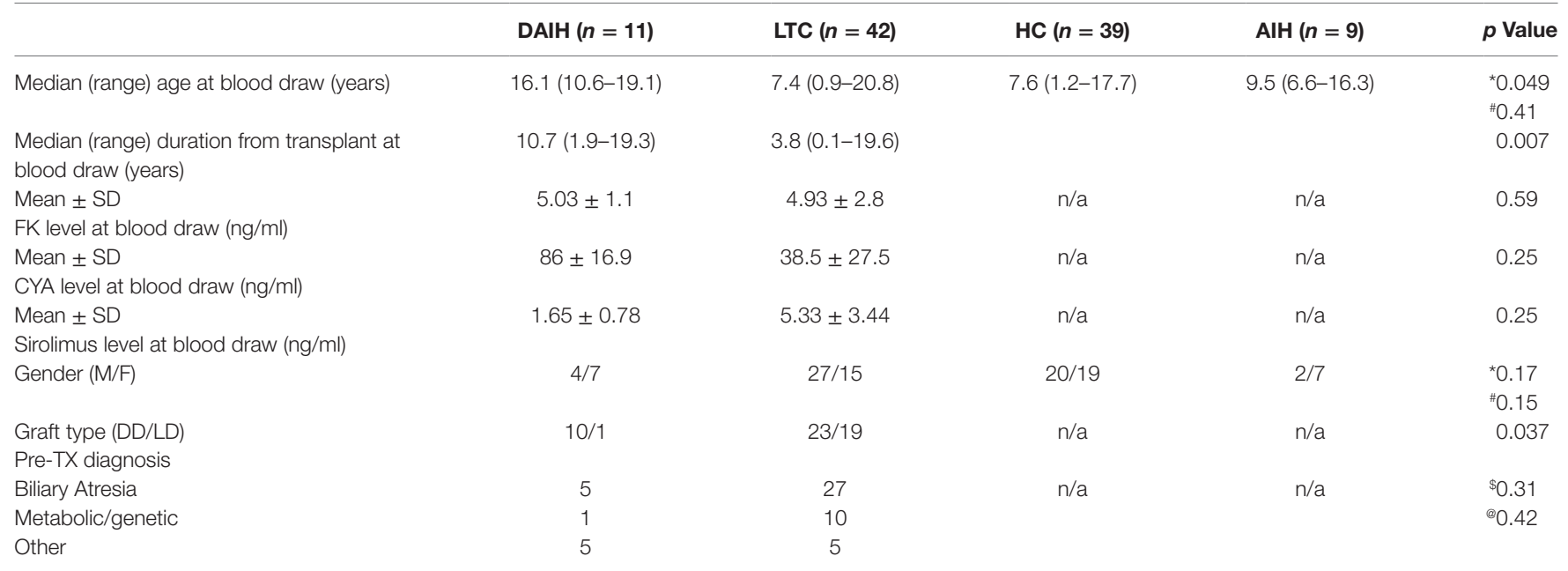

${ }^{*}$ DAIH vs. LTC.

"AlH vs. HC.

\$. Proportion of either Biliary Atresia or metabolic/genetic disease is not significantly different between DAIH vs. LTC.

KEY.

$D A I H$, de novo autoimmune hepatitis; LTC, liver transplanted control; HC, healthy control; AlH, autoimmune hepatitis; DD, deceased donor; LD, living donor; FK, tacrolimus; CYA, cyclosporin. 

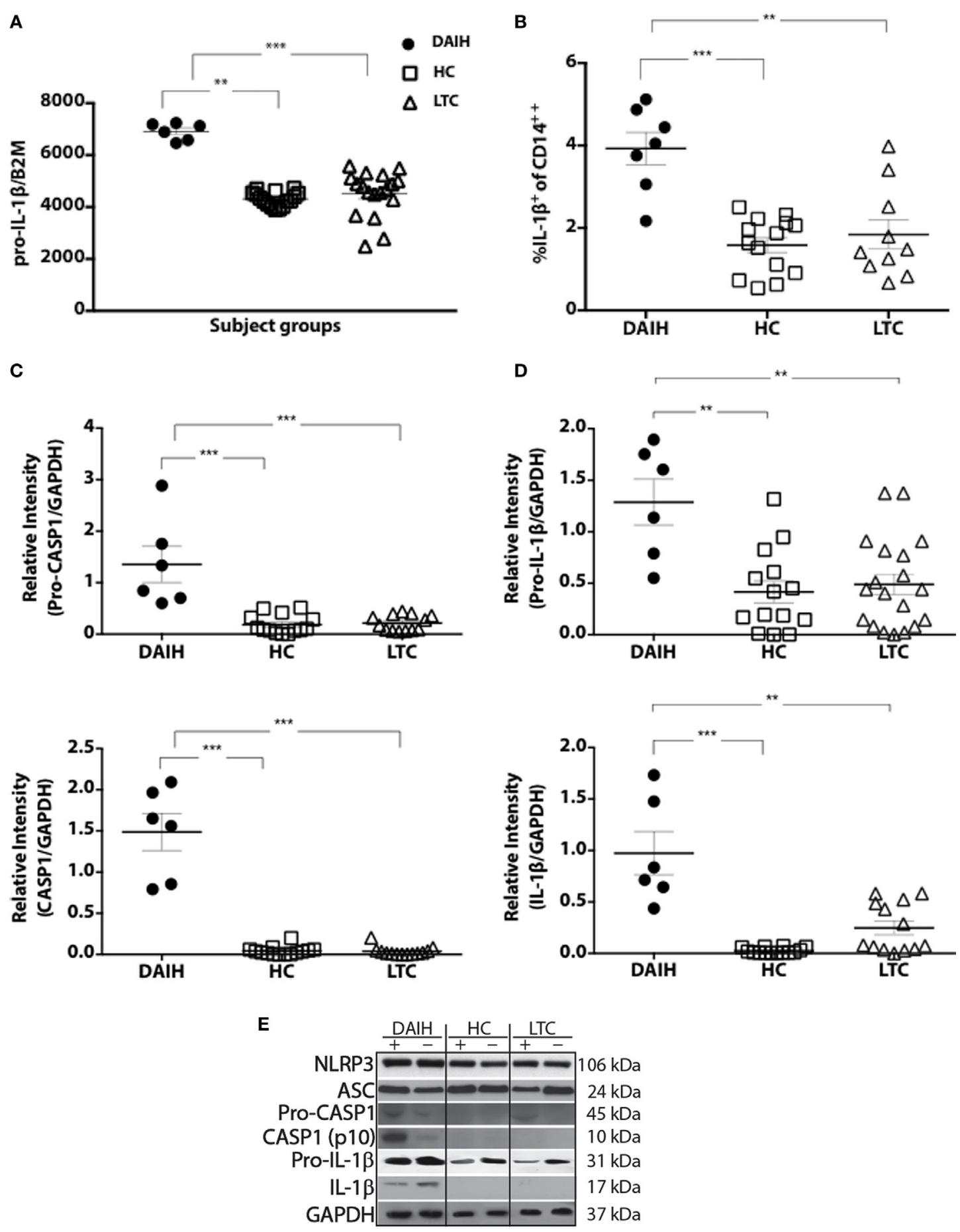

FIGURE 1 | CD14 ${ }^{++}$monocytes from liver transplanted patients with de novo autoimmune hepatitis (DAlH) undergo inflammasome activation. CD14 ${ }^{++}$monocytes from liver transplanted patients with DAIH $(n=7)$ and without DAIH (LTC) $(n=18)$ and healthy, non-transplanted children $(\mathrm{HC})(n=16)$ were stimulated with lipopolysaccharide (LPS) for $24 \mathrm{~h}$ and (i) subjected to qPCR for pro-IL-1 $\beta$; (ii) stained with anti-CD3, anti-CD14, intracellular IL-1 $\beta$. Cytokine secretion analyzed using flow cytometry; (iii) subjected to Western Blot for NLRP3, ASC, pro-caspase-1, caspase-1, IL-1 $\beta$. (A) CD14 ${ }^{++}$monocytes from patients with DAlH expressed significantly more pro- $/ L-1 \beta$ compared to CD14 ${ }^{++}$monocytes from liver transplanted patients with normal allograft function and no DAlH (LTC) ( $\left.p<0.001\right)$, as well as $\mathrm{CD}_{14}{ }^{++}$monocytes from healthy, non-transplanted children $(\mathrm{HC})(p=0.002)$. (B) CD14 ${ }^{++}$monocytes from patients with DAlH produced significantly more IL-1 $\beta$ compared to $\mathrm{CD}_{14}{ }^{++}$monocytes from liver transplanted patients with normal allograft function and no DAIH (LTC) $(p=0.005)$, as well as CD14 ${ }^{++}$monocytes from healthy, non-transplanted children $(\mathrm{HC})(p<0.001)$. (C-E) Significant pro-caspase and caspase-1 cleavage observed in CD14 ${ }^{++}$monocytes from patients with DAlH compared to CD14 ${ }^{++}$monocytes from LTC $(p<0.001 ; p<0.001)$ and HC $(p<0.001 ; p<0.001)$. Significant pro-IL-1 $\beta$ and IL-1 $\beta$ production observed in CD14 ${ }^{++}$monocytes from patients with DAlH compared to CD14 ${ }^{++}$monocytes from LTC $(p=0.007 ; p=0.002)$ and HC $(p=0.006 ; p<0.001)$. Representative blot for three patients from each subject group. Plus sign indicates with LPS stimulation, minus sign indicates absence of LPS stimulation. 
sera compared to sera of healthy children with no underlying immune-mediated disorders (HC) (Figures 2A,B). Using TLR reporter cell lines, we confirmed significantly greater activation of TLR 4 and TLR 9 by sera of patients with DAIH (compared to sera of LTC and HC subjects) (Figures 2C,D). Furthermore, protein inhibition, targeting specific DAMPs-(Fibrinogen, HSPs, and Complexes I-III), effectively reduced TLRs $2 / 4$ and 9 activation by sera of patients with DAIH (Figure 2E) (Figure S1C in Supplementary Material). Together these results are consistent with our interpretation that TLRs $2 / 4$ and 9 mediates activation of the inflammasome in $\mathrm{CD}_{14}{ }^{++}$monocytes of patients with DAIH.

Similarly, sera from children with AIH also activated TLR 2, 4, and 9 reporter cell lines (Figure S1D in Supplementary Material) further confirming that activation of the inflammasome takes place in $\mathrm{CD} 14^{++}$monocytes from patients with $\mathrm{AIH}$, and is mediated via TLRs 2, 4, and 9. The above observation supports the notion that post-transplant DAIH and $\mathrm{AIH}$ are similar diseases and may share a common disease pathogenesis. To address concerns that release of DAMPs could be related to elevated serum alanine aminotransferase (ALT) levels from hepatocyte lysis, median ALT values in subject groups were compared and no significant difference in ALT values was observed between DAIH and LTC subjects (Table S2 in Supplementary Material). Moreover, there was no significant correlation between DAMPs release and ALT for DAIH subjects (Table S3 in Supplementary Material). We also found no significant positive correlation between DAMPs and ALT in subjects with AIH; however, there was a significant negative correlation between mitochondrial DNA and ALT in subjects with AIH (Figure S1E in Supplementary Material). Taken together, the above supports our interpretation that release of DAMPs is related to disease and not hepatocyte lysis.

\section{Stimulation of TLRs 2 and 4 in CD14 ${ }^{++}$ Monocytes Promotes Th1-Like Treg Phenotype}

To confirm the role of the innate immune system in driving Treg differentiation, shRNA inhibition of TLRs $2 / 4$ and 9 in CD14 $4^{++}$ monocytes was done followed by co-culture of the monocytes with FOXP3 ${ }^{+}$Tregs as previously described (1). We confirmed shRNA inhibition of TLRs $2 / 4$ and 9 in CD $14^{++}$monocytes (Figures S2A-D in Supplementary Material). Silencing of TLRs 2 and 4 in $\mathrm{CD}_{14}{ }^{++}$monocytes resulted in significantly less IFN- $\gamma$ production by FOXP3 ${ }^{+}$Tregs (Figure 3 ; Figure S3C in Supplementary Material), however, silencing of TLR 9 did not significantly reduce IFN- $\gamma$ production by $\mathrm{FOXP}^{+}$ Tregs (Figure S3D in Supplementary Material) (gating strategy Figure S3A in Supplementary Material). The above confirms that TLR 2 and TLR 4 mediated activation of CD14 ${ }^{++}$monocytes drives Treg differentiation to pro-inflammatory, Th1-like effector Tregs in DAIH.

\section{Increased Expression of Inflammasome- Associated Components in DAlH Liver}

To test the hypothesis that inflammasome activation is also present in the liver of subjects with DAIH, CD $68^{+}$macrophages and $\mathrm{CD} 14^{++}$ monocytes were FACS sorted from liver and blood, respectively and subjected to single-cell sequencing. Absolute cell numbers varied between 126 and 9,494 cells (Table S1 in Supplementary Material). We observed that both $\mathrm{CD} 14^{++}$monocytes (blood) and $\mathrm{CD}^{+} 8^{+}$macrophages (liver) obtained from patients with DAIH clustered together and away from monocytes and macrophages obtained from liver transplanted subjects with normal allograft function (LTC) suggesting that $\mathrm{CD}_{14}{ }^{++}$monocytes and $\mathrm{CD} 68^{+}$ macrophages obtained from subjects with DAIH are indeed very similar in terms of their transcriptional profile (Figure 4A). Upon closer examination of individual clusters of cells, the cells within clusters 3 and 4 contained $\mathrm{CD} 14^{++}$monocytes and $\mathrm{CD}^{+} 8^{+}$ macrophages from subjects with DAIH while cells within cluster 6 contained $\mathrm{CD} 14^{++}$monocytes and $\mathrm{CD}^{+} 8^{+}$macrophages from LTC subjects (Figures S4A,B in Supplementary Material). We then proceeded to compare clusters $3+4$ vs. cluster 6 . There were 135 genes significantly overexpressed in the clusters $3+4$ vs. cluster 6 comparison and 258 genes significantly overexpressed in the cluster 6 vs. clusters $3+4$ comparison (Figure S4C in Supplementary Material). Some of the significantly expressed genes in clusters $3+4$ cells (i.e., DAIH monocytes/macrophages) compared to cluster 6 cells (i.e., LTC monocytes/macrophages) include HMGB1, which provides inflammatory signal 1 to TLR 4 in the two-signal activation of the inflammasome machinery (3), CARD19, the caspase recruitment domain (CARD) is present in death-domain superfamily proteins and involved in inflammation and apoptosis (7). Importantly, the inflammasome complex includes a CARD containing protein (8), and TIMP1 (Figure 4B). In the cluster 6 vs. clusters $3+4$ comparison, tumor necrosis factor $\alpha$-induced protein 3 (TNFAIP3), a negative regulator of the NLRP3 inflammasome (9), was significantly expressed in cluster 6 (i.e., LTC monocytes/ macrophages) and almost absent in clusters $3+4$ (i.e., DAIH monocytes/macrophages) (Figures 4C,D). We believe that these observations support a role for inflammasome activation in disease pathogenesis of DAIH. To support our interpretation of common features in underlying pathogenesis of both DAIH and $\mathrm{AIH}$, we subjected $\mathrm{CD} 14^{++}$monocytes of non-transplanted subjects with AIH to single-cell RNA-sequencing and observed 1 cluster of AIH cells that appeared associated with the DAIH cluster of cells; additionally, some AIH cells intermingled with DAIH cells, however, this percentage was low (Figure S4D in Supplementary Material). Upon closer examination of individual cluster of cells, cluster 3 has AIH cells and cluster 11 has cells from liver transplanted subjects with normal allograft function who do not have DAIH (LTC) (Figure S4E in Supplementary Material); specifically, cluster 3 has $90 \%$ proportion of AIH cells, $10 \%$ proportion of DAIH cells while cluster 11 has $90 \%$ proportion of LTC cells (Figure S4F in Supplementary Material). We therefore proceeded to compare cluster 3 vs. cluster11. TIMP1 and the heat shock family of proteins were significantly overexpressed in the cluster 3 cells vs. cluster 11 comparison: TIMP1: average $\log \mathrm{FC} 2.33 p=9.0 \times 10^{-223}$; HSP90: average $\log \mathrm{FC} 0.4 p=1.15 \times 10^{-95}$ (Figure S4G in Supplementary Material). We believe the above supports a role for inflammasome activation in disease pathogenesis of AIH.

The most important advantage of single-cell RNA-sequencing is the ability to study transcriptomes of populations of cells. Unlike FACS that solely depends on surface markers, single-cell RNA-seq 
allows us to study the heterogeneity in the population of cells that might have identical cell surface markers. As our samples are from different patients and thus have an $n$ that is greater than 1 , they are not technical replicates Therefore, even small populations of cells that show specific transcription profiles can still provide statistically robust and biologically meaningful results.

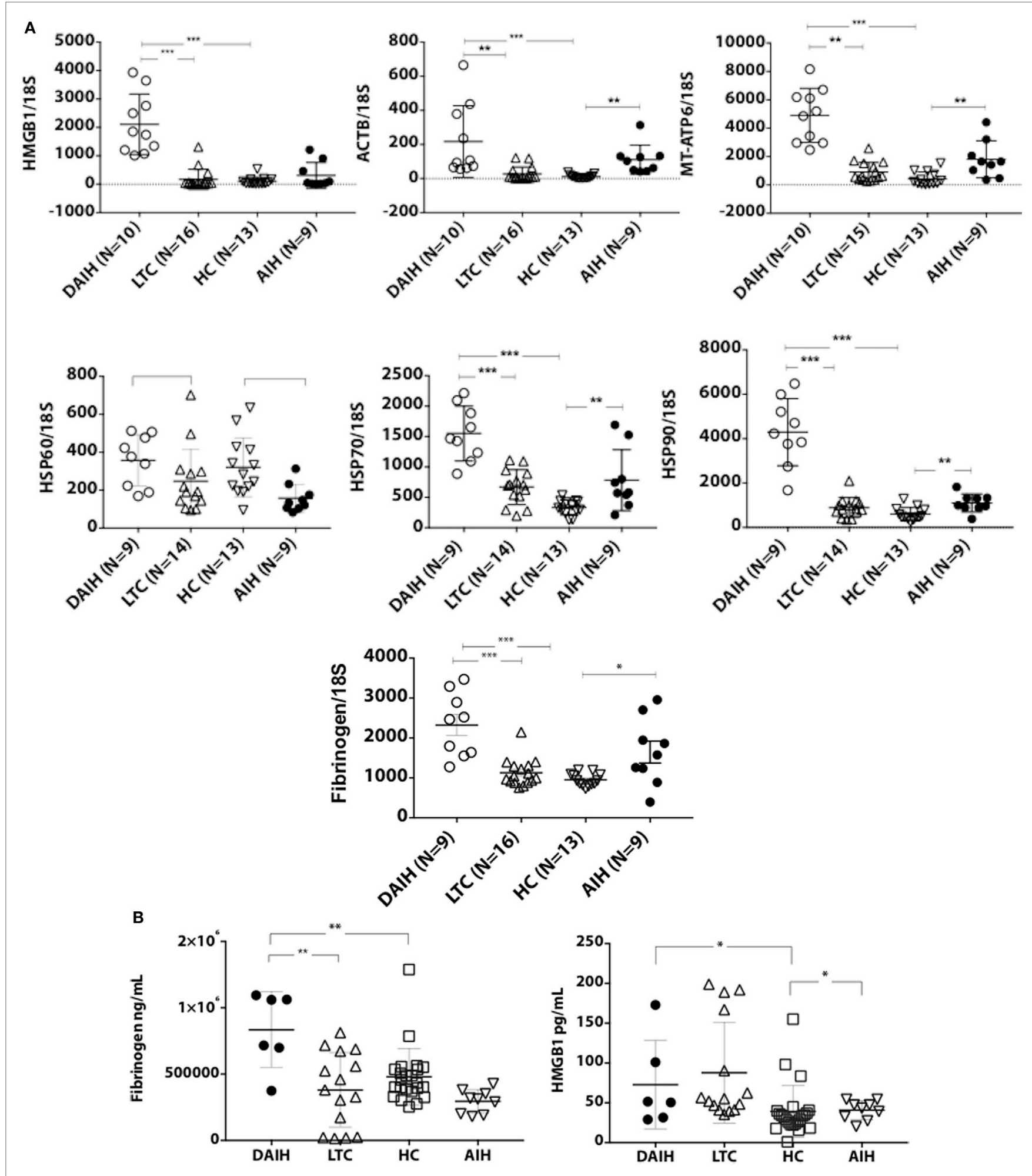

FIGURE 2 | Continued 


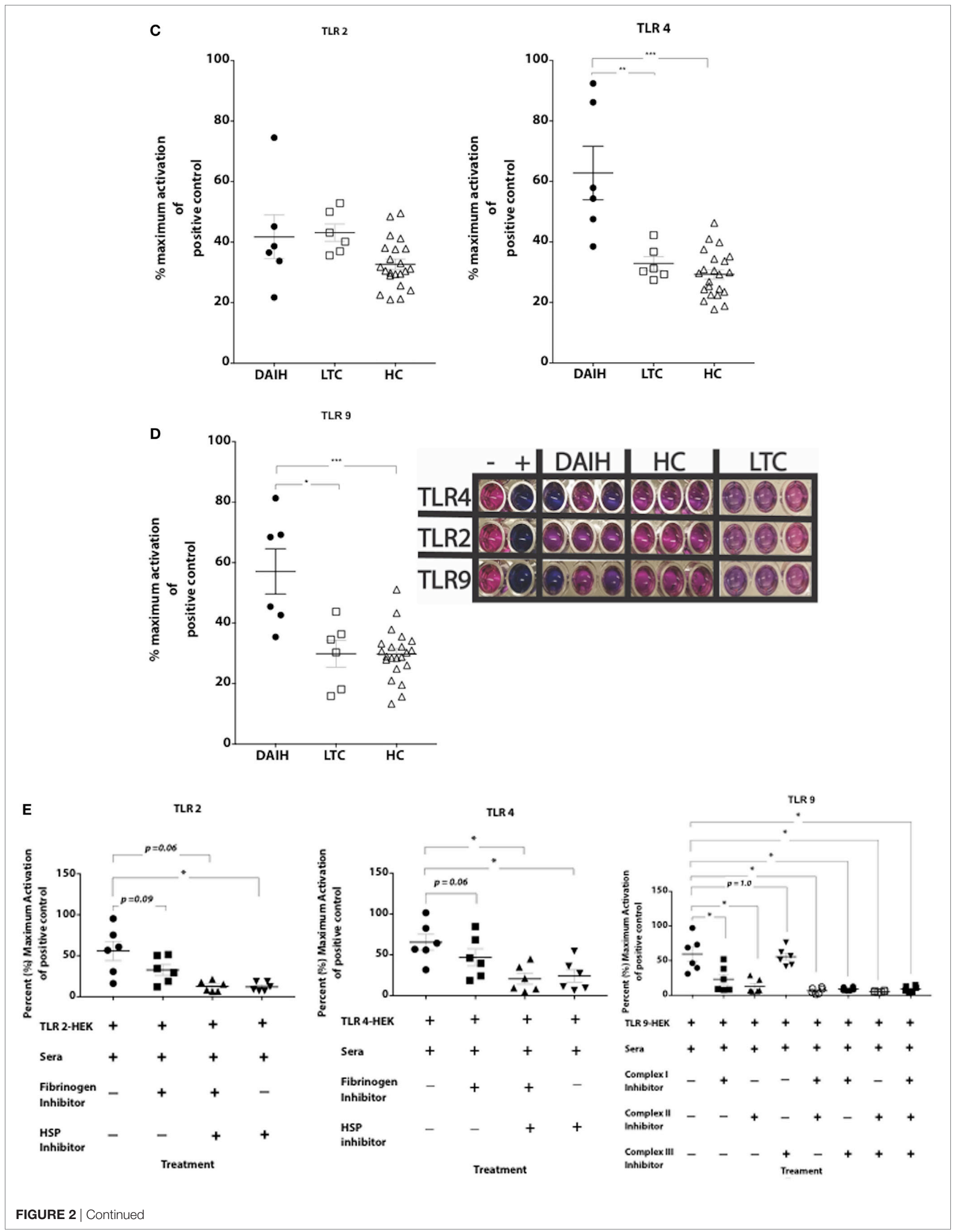


FIGURE 2 | Damage-associated molecular patterns (DAMPs) providing inflammatory signal to the receptors toll-like receptor (TLR) 2/4 and 9, are significantly increased in sera of patients with de novo autoimmune hepatitis (DAlH). Sera from liver transplanted patients: with DAlH $(n=10)$, without DAIH (LTC) $(n=19)$; healthy, non-transplanted children $(\mathrm{HC})(n=29)$, and non-transplanted children with autoimmune hepatitis (AlH) $(n=9)$, was subjected to (i) qPCR; (ii) ELISA. HEK cells that stably co-express a human TLR2, TLR4, or TLR9 gene and an NF-kB-inducible secreted embryonic alkaline phosphatase (SEAP) reporter gene were used to determine activation of TLRs from patient sera. (A,B) significant abundance of DAMPs that activate TLRs $2 / 4$ and 9 and levels of cytosolic proteins associated with TLR $2 / 4$ activation in sera of patients with DAlH compared to sera of LTC subjects (qPCR: $p<0.001$ for ACTB; $p<0.001$ for MT-ATP/complex I; $p<0.001$ for Fibrinogen; $p<0.001$ for HMGB1, $p<0.001$ for HSP70; and $p<0.001$ for HSP90, $p=0.05$ for HSP60) (ELISA: $p=0.003$ for Fibrinogen; $p=0.37$ for HMGB1). Significant abundance of DAMPs that activate TLRs $2 / 4$ and 9 and levels of mitochondrial, cytosolic, and functional proteins in sera of patients with DAlH compared to sera of HC subjects (qPCR: $p<0.001$ for Fibrinogen, HMGB1, HSP70, HSP90, ACTB, and MT-ATP6, respectively, and $p=0.64$ for HSP60). Significant abundance of DAMPs that activate TLRs $2 / 4$ and 9 and levels of functional proteins in sera of patients with AlH compared to sera of HC subjects. [qPCR: $p<0.001$ for ACTB (nuclear DNA); $p=0.002$ for MT-ATP6 (mitochondrial DNA); $p=0.013$ for Fibrinogen; $p=0.008, p=0.005$, and $p=0.008$ for HSPs 60,70 and 90 , respectively] (ELISA: $p=0.043$ for HMGB1; $p=0.11$ for fibrinogen). ${ }^{\star * *} p<0.001,{ }^{* *} p<0.01$, ${ }^{*} p<0.05$. (C,D) Activation of TLRs 2,4 , and 9 reporter cell lines by sera of patients with DAlH. DAlH vs. LTC: $(p=0.47$; TLR2) $(p=0.008 ;$ TLR4) $(p=0.02 ;$ TLR9). DAlH vs. HC: $(p=0.17$; TLR2) $(p<0.001 ;$ TLR4) $(p=0.001 ;$ TLR9). Minus sign: negative control, plus sign: positive control. Representative plate for three patients from each subject group and summary data. (E) Protein inhibition targeting heat shock protein, fibrinogen, and complexes I-III significantly reduced activation of TLR2/4 and TLR9 reporter cell lines, respectively [for TLR 9 reporter cell line inhibition: $p=0.03$ for Rotenone (Complex 1), $p=0.03$ for Thenoyltrifluoroacetone, TTFA (Complex II), $p=1.0$ for Antimycin A (Complex III), $p=0.03$ for Complexes I and II, $p=0.03$ for Complexes I and III, $p=0.03$ for Complexes II and III, $p=0.03$ for Complexes I, II, and III; for TLR 4 reporter cell line inhibition: $p=0.06$ for fibrinogen inhibitor, $p=0.03$ for the HSP90 inhibitor, 17-N-Allylamino-17-demethoxygeldanamycin, $17 \mathrm{AAG}$, and $p=0.03$ for both HSP90 and fibrinogen inhibitors; for TLR 2 reporter cell line inhibition: $p=0.09$ for Fibrinogen inhibitor, $p=0.03$ for the HSP90 inhibitor, $17 \mathrm{AAG}, p=0.06$ for both HSP90 and fibrinogen inhibitors]. (HSP-heat shock protein; positive controls: FSL-1 - synthetic diacylated lipoprotein, LPS - lipopolysaccharide, ODN 2006-CpG oligonucleotide). Summary data.

\section{DAlH Treg: TLR 2 scramble control RNA treated monocyte}

\section{DAIH Treg: TLR 2 shRNA treated monocyte}
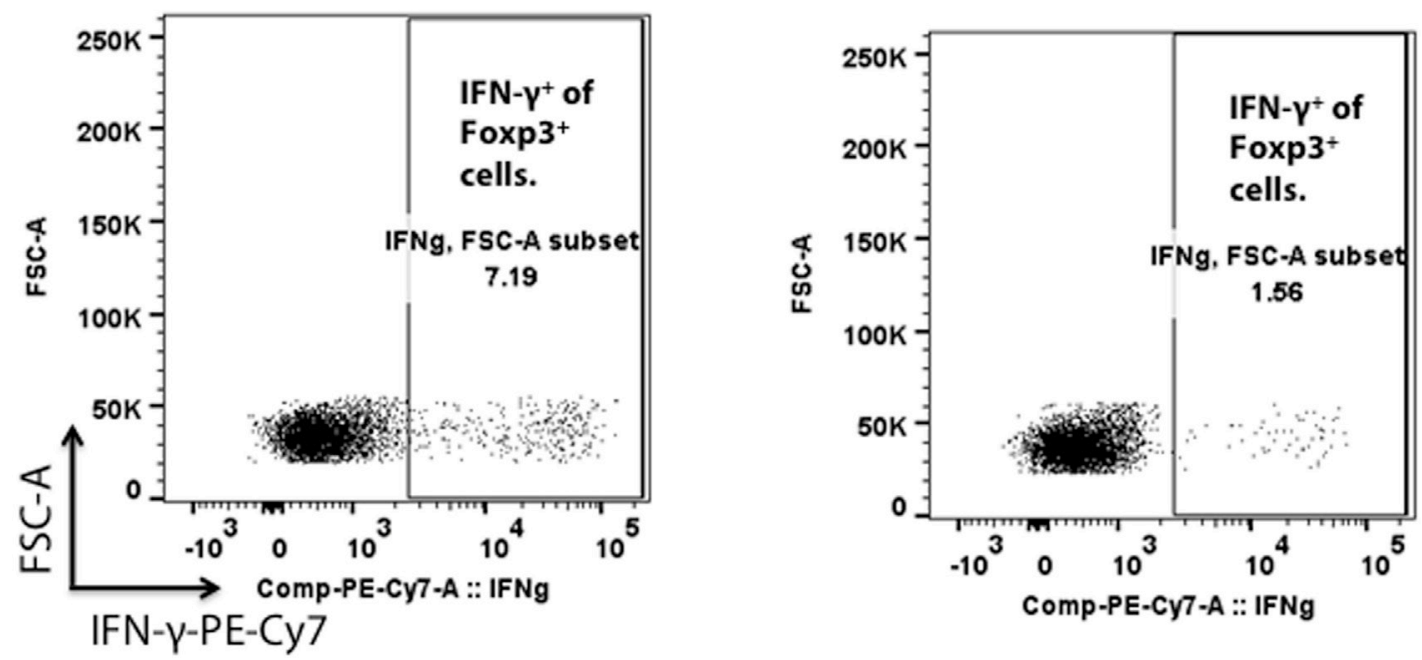

FIGURE 3 | Silencing of toll-like receptors (TLRs) 2 and 4 in CD14++ monocytes of patients with de novo autoimmune hepatitis (DAIH) prevents IL-12-mediated regulatory T cell (Treg) differentiation to Th1-like Tregs. CD14++ monocytes from patients with DAlH $(n=4)$ were subjected to shRNA inhibition of TLR $2 / 4$ and 9 and then co-cultured with sorted $\mathrm{CD} 4{ }^{+} \mathrm{CD} 25^{\mathrm{h}} \mathrm{CD} 127^{\text {neg }} \mathrm{FOXP} 3^{+}$Tregs from patients with DAlH and $\mathrm{HC}$ subjects, in the presence of plate bound anti-CD3 for 5 days and IFN- $\gamma$ production from FOXP3 $3^{+}$Tregs was assessed using flow cytometry. Representative flow cytometry plot showing reduced IFN- $\gamma$ production from FOXP3 ${ }^{+}$Tregs of DAlH patients following co-culture of their sorted Tregs with their silenced TLR 2 monocytes and scramble control monocytes.

\section{Hepatocyte Apoptosis Is Induced by CD14 ${ }^{++}$Monocytes}

In addition to driving Treg differentiation, we sought to determine if the histological inflammation observed in DAIH is induced by $\mathrm{CD} 14^{++}$monocytes. To this end, $\mathrm{CD} 14^{++}$monocytes from PBMCs were co-cultured over $96 \mathrm{~h}$ with normal human hepatocytes. The readout was anti-caspase- 3 from hepatocytes and alanine aminotransferase secretion in culture supernatant. Hepatocytes cultured alone did not induce hepatocyte apoptosis at $24 \mathrm{~h}$
(Figure S5A in Supplementary Material). After 96 h of hepatocyte co-culture with $\mathrm{CD} 14^{++}$monocytes obtained from subjects with DAIH, we observed hepatocyte apoptosis (Figure 5A), more so than following co-culture with $\mathrm{CD} 14^{++}$monocytes obtained from LTC subjects (Figure 5B). This induction of hepatocyte apoptosis at $96 \mathrm{~h}$ by $\mathrm{CD} 14^{++}$monocytes from subjects with DAIH was accompanied by elevation of alanine aminotransferase in the culture supernatant (Figure S5C in Supplementary Material). CD $14^{++}$monocytes from LTC subjects and healthy children did 


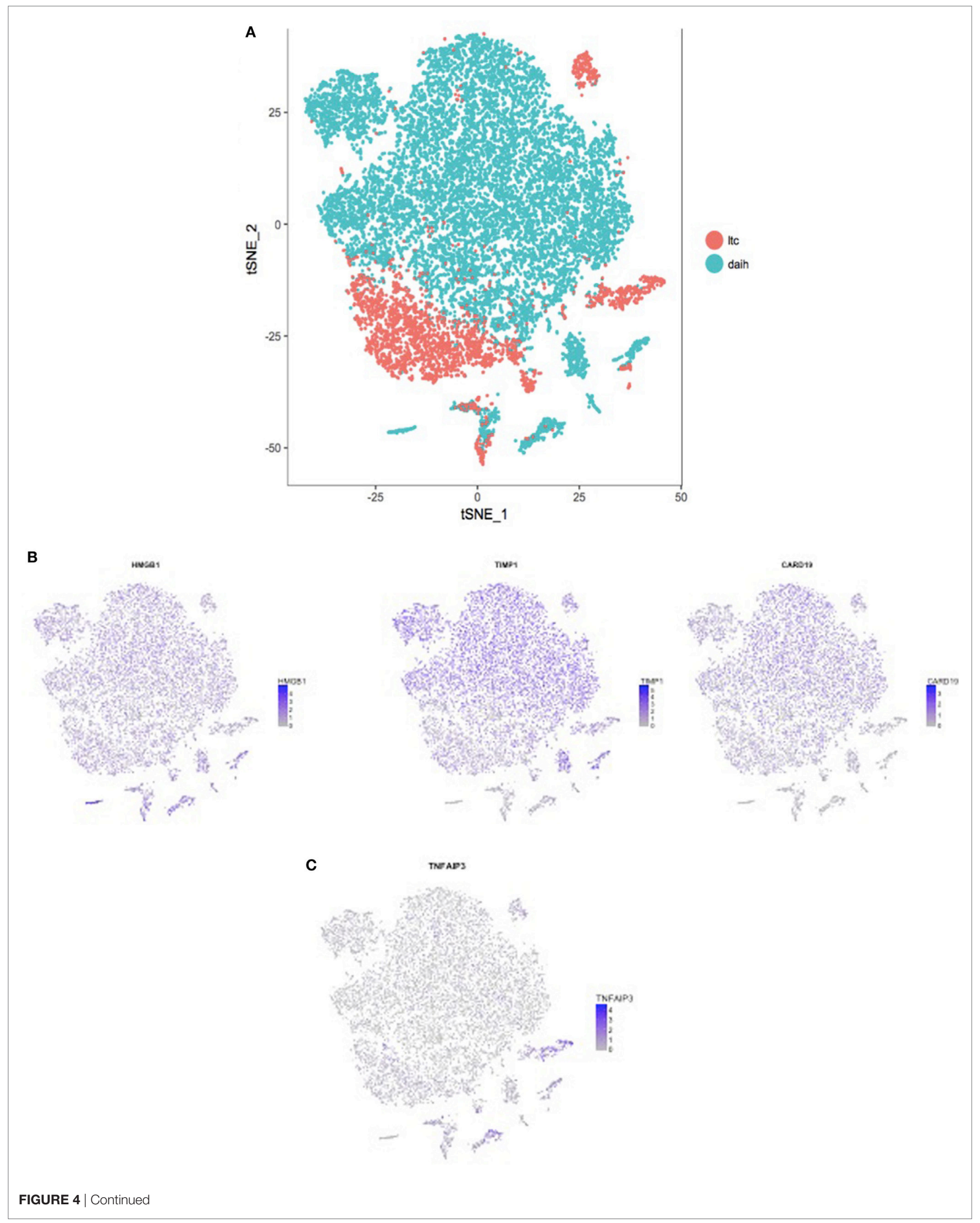




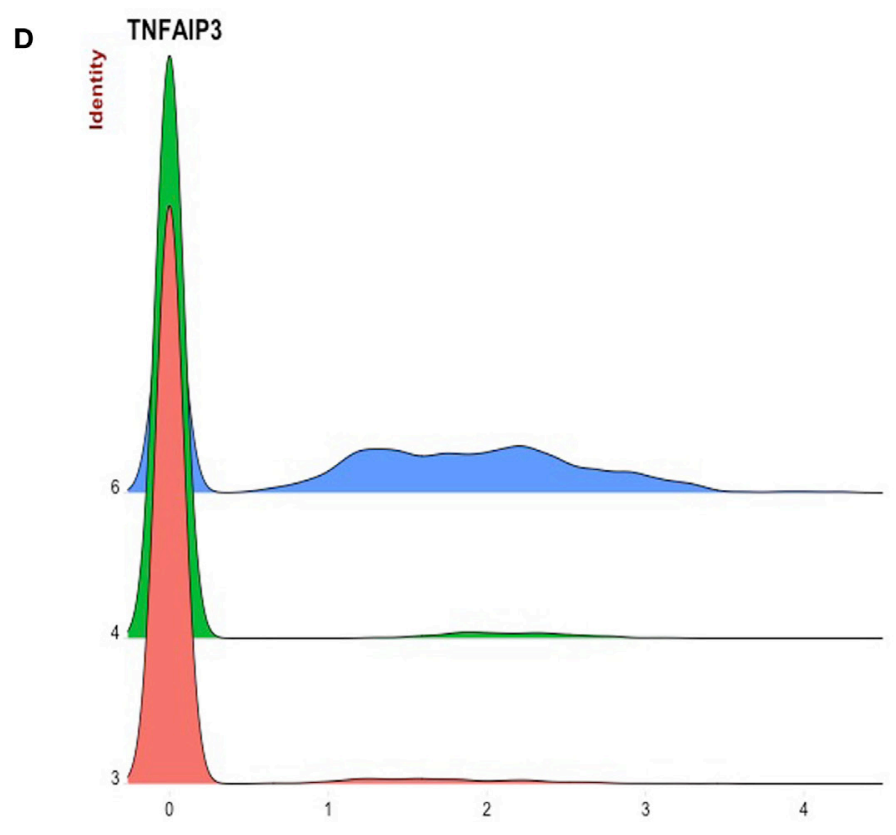

FIGURE 4 | CD14++ monocytes from peripheral blood mononuclear cells (PBMCs) and CD68 ${ }^{+}$macrophages from the liver of patients with de novo autoimmune hepatitis (DAlH) display increased expression of inflammasome-associated components and absence of a negative regulator of infammasome activation. PBMCs from blood and intrahepatic lymphocytes from the liver were obtained from liver transplanted patients with DAlH ( $n=5)$, and liver transplanted patients with normal allograft function who do not have DAIH (LTC) $(n=4)$ ), stained for CD3CD14 (PBMC) and CD45CD68 (intrahepatic lymphocytes), and the monocyte (CD3-CD14 ${ }^{++}$PBMC) and macrophage (CD45+CD68+ liver) population were then sorted on a FACS Aria and subjected to library preparation for single-cell sequencing. After log normalizing and scaling the data, variable genes were detected using Seurat (10). These highly variable genes were used to generate principal components, and cluster detection was done using Seurat. The clusters were visualized using the tSNE rendering in Seurat. (A) CD14 ${ }^{++}$monocytes and CD68 ${ }^{+}$macrophages from subjects with de novo autoimmune hepatitis cluster together and away from monocytes and macrophages of LTC subjects. (B) After cluster detection, marker genes for each cluster were detected such that the gene(s) is expressed in at least $25 \%$ of the cells in the given cluster, and it is overexpressed by at least $25 \%$ than all of the rest of the cells. The expression values for these genes were then plotted in each cell, and rendered according to the tSNE to give the feature plots. Feature plots showing significant overexpression of $H M G B 1$ $\left(p=2.62 \times 10^{-69}\right.$, fold $\left.>3\right)$, CARD19 $\left(p=2.13 \times 10^{-59}\right.$, fold $\left.>2\right)$, and TIMP1 $\left(p=1.03 \times 10^{-79}\right.$, fold $\left.>3\right)$ in clusters $3+4$ compared to cluster 6 i.e., significant overexpression in monocytes/macrophages of subjects with DAlH compared to monocytes/macrophages of LTC subjects. (C) Feature plot showing significant overexpression of tumor necrosis factor $\alpha$-induced protein 3 (TNFAIP3) $\left(p=3.44 \times 10^{-233}\right.$, fold $>2$ ) in cluster 6 compared to clusters $3+4$ i.e., significant overexpression in monocytes/macrophages of LTC subjects compared to monocytes/macrophages of subjects with DAlH. (D) Joy plot confirms the near absence of TNFAIP3 from monocytes/macrophages of patients with DAIH and its high expression in monocytes/macrophages of LTC subjects.

not induce an elevation in alanine aminotransferase in culture supernatant following 72 and $96 \mathrm{~h}$ of co-culture with hepatocytes (Figure S5C in Supplementary Material). This result suggests $\mathrm{CD}_{14}{ }^{++}$monocytes from subjects with DAIH may cause select forms of hepatocyte injury. Perhaps not surprisingly, CD14 $4^{++}$ monocytes obtained from non-transplanted subjects with $\mathrm{AIH}$ also induced hepatocyte apoptosis following a 96-h co-culture period (Figure 5C), however, this was not accompanied by elevations in alanine aminotransferase in culture supernatant (Figure S5C in Supplementary Material). As expected, CD14 ${ }^{++}$ monocytes from healthy children did not induce hepatocyte apoptosis (Figure S5B in Supplementary Material).

These results lead us to propose the following as the underlying immune pathogenesis in DAIH: DAMPs activate TLR2, TLR4 and TLR9 in $\mathrm{CD}_{1} 4^{++}$monocytes promoting inflammasome activation with inflammatory cytokine secretion, including IL-12, from CD14 ${ }^{++}$monocytes. IL-12 drives Treg differentiation with a resultant dramatic increase in IFN- $\gamma$ secretion from Tregs. These IFN- $\gamma$ secreting Tregs (also called
Th1-like Tregs) have previously been shown to lack the ability to suppress $\mathrm{T}$ effector cell proliferation $(1,11,12)$. Inhibition of TLR2 and TLR4 (but not TLR 9) activation abrogates secretion of IL-12 thus preventing differentiation of Tregs to IFN- $\gamma$ secreting Tregs (Figure 6).

\section{DISCUSSION}

The main finding in our study is that TLRs 2- and 4-mediated inflammasome activation in $\mathrm{CD}_{1} 4^{++}$monocytes is key to maintaining dysfunctional Tregs in liver transplanted patients with DAIH. Our study is the first to relate inflammasome activation in $\mathrm{CD}_{14}{ }^{++}$monocytes to Treg dysfunction in DAIH. The inflammasome complex is a critical mediator in various autoimmune diseases; Moreover, it is thought to be a pivotal pathway connecting tissue injury with inflammation and autoimmunity (13). This is elegantly demonstrated in mice with Abcal/g1 deficiency in dendritic cells (DCs) that have a lupus-like phenotype. Their $\mathrm{CD} 11 \mathrm{~b}^{+} \mathrm{DC}$ population 

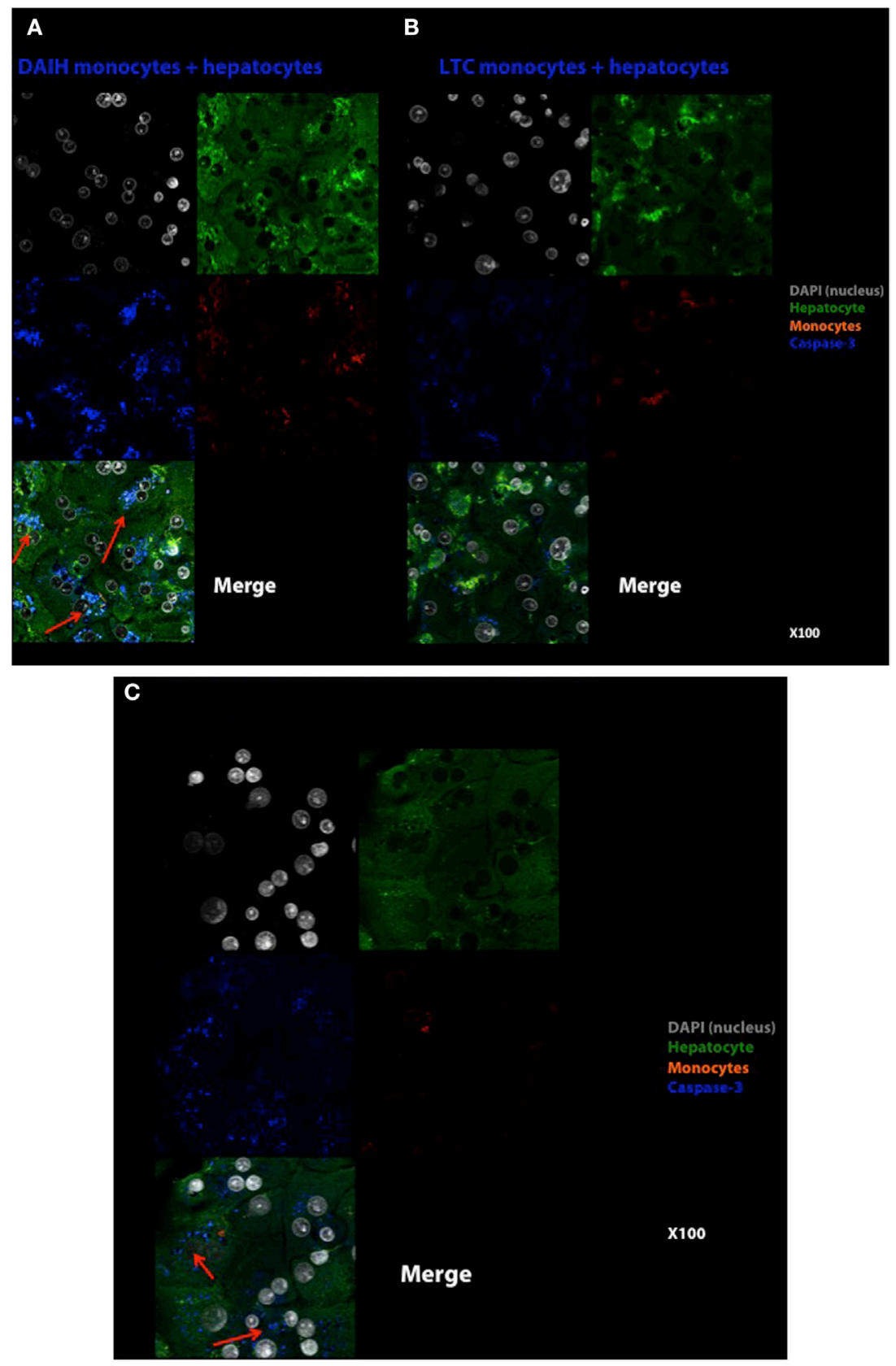

FIGURE 5 | CD14 ${ }^{++}$monocytes from patients with de novo autoimmune hepatitis (DAIH) induce hepatocyte perturbation as evidenced by caspase-3 cleavage of hepatocytes. CD14 ${ }^{++}$monocytes from liver transplanted patients with DAlH $(n=2)$, liver transplanted patients with normal allograft function who do not have DAlH (LTC) $(n=2)$, and non-transplanted children with autoimmune hepatitis (AlH) $(n=2)$ were isolated by negative selection and co-cultured with normal hepatocytes over $96 \mathrm{~h}$, and anti-active caspase-3 production by hepatocytes were assessed by confocal microscopy. (A) Hepatocytes cultured with DAlH monocytes for $96 \mathrm{~h}$. DAlH monocytes induce hepatocyte apoptosis (arrows). Top left: DAPI (nucleus) - gray, top right: hepatocytes - green, middle left: caspase-3-blue, middle right: monocytes - orange, bottom left: merge. 100x magnification. (B) Hepatocytes cultured with LTC monocytes for 96 h. Top left: DAPI (nucleus) - gray, top right: hepatocytes - green, middle left: caspase-3-blue, middle right: monocytes-orange, bottom left: merge. 100x magnification. (C) Hepatocytes cultured with AlH monocytes for 96 h. AlH monocytes induce hepatocyte apoptosis (arrows). Top left: DAPI (nucleus) - gray, top right: hepatocytes - green, middle left: caspase-3blue, middle right: monocytes - orange, bottom left: merge. 100× magnification.

displays inflammasome activation and enhanced secretion of the inflammatory cytokines, IL-1 $\beta$ and IL-18, leading to skewing of T cells toward a Th1 phenotype. Deficiency of the NOD-like receptor family pyrin domain containing 3 (NLRP3) partly reversed some of the lupus-like features, as well as the increase in IL- $1 \beta$ and Th1 expansion indicating that this pathway contributes to aspects of the autoimmunity phenotype in DC-Abcal/g1 deficiency(14). 
Model of Reduced Suppressive Function of Tregs and Th1-like Treg Development after TLR2/4 Stimulation

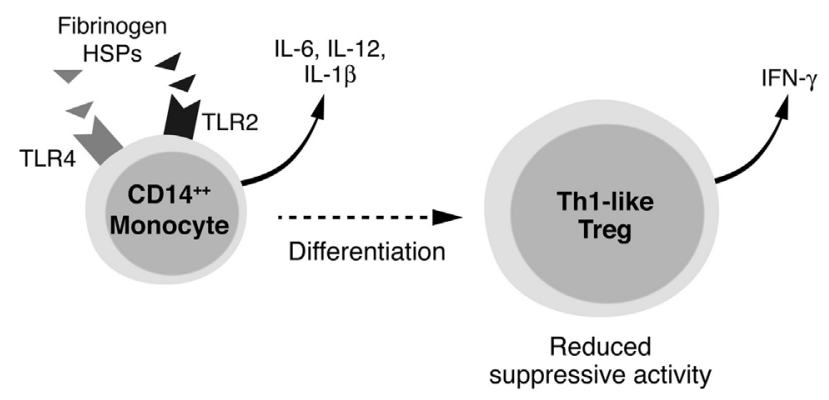

FIGURE 6 | Conceptual framework for immune pathogenesis of de novo autoimmune hepatitis (DAIH). Damage-associated molecular patterns (DAMPs) in sera of patients with DAlH activate toll-like receptors (TLRs) 2, 4, and 9 in $\mathrm{CD}_{14}{ }^{++}$monocytes, leading to activation of the inflammasome, caspase- 1 cleavage, pro-IL-1 $\beta$ processing to IL-1 $\beta$ and IL-1 $\beta$ release. Activation of monocytes is also accompanied by production of other inflammatory cytokines including $\mathrm{IL}-12$. In the presence of regulatory $T$ cells (Tregs), IL-12 drives Tregs to secrete IFN- $\gamma$ and thus become Th1-like Tregs that lack suppressive function (1, 11, 12). Inhibition of TLR 2 and TLR 4 (but not TLR 9) prevents secretion of IL-12 and thus prevents differentiation of Tregs to Th1-like Tregs.

In agreement with Westerterp et al. (14), our current work demonstrates that $\mathrm{CD} 14^{++}$monocytes of patients with DAIH display inflammasome activation mediated via TLR2 and TLR4, with enhanced IL-12 secretion that drives IFN- $\gamma$ secretion from Tregs and Treg dysfunction. TLR2 and TLR4 silencing in CD14 $4^{++}$ monocytes of these patients prevents Treg differentiation to Th1like Tregs and thus IFN- $\gamma$ secretion by Tregs of these patients (Figure 3), suggesting that autoimmunity is promoted by $\mathrm{CD} 14^{++}$ monocytes predominantly through activation of inflammatory signaling pathways.

Further support for involvement of inflammasome activation in the pathogenesis of DAIH is illustrated by sera from patients with DAIH activating TLR2, TLR4, and TLR9 reporter cell lines (Figures 2C,D), and protein inhibition targeting specific DAMPs effectively reducing this activation of TLR reporter cell lines (Figure 2E), indicating an essential contribution of the inflammasome to key aspects of the autoimmune phenotype in DAIH. Moreover, we observed significantly increased expression of HMGB1 by $\mathrm{CD}^{+} 8^{+}$macrophages of subjects with DAIH (Figure 4B). TLR 4 appears to be the dominant receptor for HMGB1-mediated activation of macrophages (15). Direct involvement of inflammasomes in autoimmunity has been described in vitiligo $(16,17)$, systemic lupus erythematosus (SLE) $(18,19)$, and experimental autoimmune encephalomyelitis $(20,21)$. In addition, IL-18, the other effector cytokine of inflammasome-mediated caspase-1 activation has been implicated in the dysfunction of endothelial progenitor cells in SLE, impairing vascular repair (22). IL-18 also contributes to secondary progressive multiple sclerosis and rheumatoid arthritis joint inflammation, stimulating leukocyte chemotaxis, angiogenesis and cartilage destruction $(23,24)$.

Both priming and activation of inflammasomes require tight regulation to prevent an excessive inflammatory response.
Inflammasome pathways are negatively regulated at multiple levels. Recently, A20/TNFAIP3 was found to be a negative regulator of the NLRP3 inflammasome, and specific deletion of A20 in myeloid cells resulted in spontaneous arthritis in mice that resembled human rheumatoid arthritis (9). We have demonstrated significantly increased expression of TNFAIP3 in monocytes/macrophages of liver transplanted subjects without DAIH who have normal allograft function (LTC) (Figures 4C,D), and whose monocytes do not undergo inflammasome activation (Figures 1C-E) further supporting direct involvement of the inflammasome in pathogenesis of DAIH.

We questioned if the inflammation observed histologically in patients with DAIH is induced in part by their $\mathrm{CD}_{1} 4^{++}$monocytes. To overcome the limitation of the lack of an animal model of DAIH, we co-cultured CD $14^{++}$monocytes of patients with DAIH with normal human hepatocytes to observe if these monocytes could change hepatocyte survival. Following $96 \mathrm{~h}$ of co-culture, CD $14^{++}$monocytes from patients with DAIH induced apoptosis of normal hepatocytes (Figure 5A). The induction of apoptosis was accompanied by an increase in alanine aminotransferase levels in the culture supernatant (Figure S5C in Supplementary Material). Interestingly, even though $\mathrm{CD} 14^{++}$monocytes from non-transplanted subjects with $\mathrm{AIH}$ also induced hepatocyte apoptosis at $96 \mathrm{~h}$ (Figure 5C), it was not accompanied by alanine aminotransferase elevation (Figure S5C in Supplementary Material). This could be due to the fact that in $\mathrm{AIH}$, a more prolonged co-culture period may be necessary to demonstrate accompanying liver enzyme elevation in the culture supernatant. Alternatively, it is also possible that their current treatment may result in normalization of liver biochemistry but not complete normalization of histological activity. Hepatocyte injury as a consequence of $\mathrm{CD} 14^{++}$monocyte actions has been reported by Nishio et al. (25) who observed that CD14 ${ }^{++}$monocyte derived galectin- 9 increasing the toxicity of natural killer cells in chronic hepatitis $\mathrm{C}$, with resultant liver injury and persistent infection.

There are several limitations to our study; first, we performed the TLR silencing experiments using $\mathrm{CD} 14^{++}$monocytes from blood and not $\mathrm{CD}^{+} 8^{+}$macrophages from the liver However, there is an enhanced ability of monocytes to enter the liver from blood through hepatic endothelium as reported by Liaskou et al. who show monocyte transendothelial migration across inflamed hepatic sinusoidal endothelium in vitro (26). In addition, monocytes circulate in blood for several days before migrating into tissues where they can differentiate into tissue macrophages (27). Co-expression of CD14, CD16, and CD68 on a population of large cells with the morphology of macrophages is present throughout the liver sinusoids confirming that distinct monocyte-derived and sessile macrophage populations coexist in the liver (26). Moreover, we have previously demonstrated co-expression of $\mathrm{CD} 14^{+} \mathrm{CD} 68^{+}$cells in the liver of patients with DAIH confirming co-existence of monocyte-derived and tissue macrophages in the liver as well as suggesting that these monocytes are recruited from the blood (1). Second, our findings could be related to having a transplanted allograft and not necessarily the underlying disease. However, we have shown the absence of TLR 2 and 4-mediated inflammasome activation in $\mathrm{CD} 14^{++}$monocytes of similarly liver transplanted subjects who do not have DAIH. Furthermore, 
CD $14^{++}$monocytes of non-transplanted subjects with AIH also undergo TLRs 2, 4, and 9-mediated inflammasome activation supporting our contention of involvement of inflammasome activation in the pathogenesis of DAIH. Moreover, the biochemical (presence of autoantibodies and immunoglobulin G), histological features (peri-portal lympho-plasmacytic inflammation with interface hepatitis), and treatment of AIH and DAIH are the same. Third, the lack of a suitable animal model of DAIH has precluded us from demonstrating our findings in vivo. It is possible that co-culture of DAIH CD14 ${ }^{++}$monocytes + Tregs with hepatocytes for a prolonged time period may result in even more hepatocyte perturbation with aminotransferase elevation than we have demonstrated, perhaps similar to that seen in the serum of untreated subjects with DAIH or that co-culture of these monocytes + Tregs with cholangiocytes may result in bile duct injury similar to that reported in subjects with DAIH (4, 28). If this is clearly demonstrated, then addressing prevention of inflammasome activation either by inhibition of TLR 2 and TLR 4 or protein inhibition targeting specific DAMPs would need to be investigated in humans.

\section{CONCLUSION}

Priming of inflammasomes is increased in both DAIH and AIH due to innate immune stimuli released from damaged cells. Understanding the regulatory network of inflammasome activation in these two diseases will shed light on the development of new target therapy. In addition, large population genetic association studies looking at single-nucleotide polymorphisms in inflammasome components in these two diseases would be helpful to validate our current data.

\section{ETHICS STATEMENT}

This study was carried out in accordance with the recommendations of [Yale Institutional Review Board], [Human Investigation Committee]. The protocol was approved by the

\section{REFERENCES}

1. Arterbery AS, Osafo-Addo A, Avitzur Y, Ciarleglio M, Deng Y, Lobritto S, Martinez M, et al. Production of proinflammatory cytokines by monocytes in liver-transplanted recipients with de novo autoimmune hepatitis is enhanced and induces TH1-like regulatory T cells. J Immunol (2016) 196:4040-51. doi:10.4049/jimmunol.1502276

2. Bauernfeind F, Ablasser A, Bartok E, Kim S, Schmid-Burgk J, Cavlar T, et al. Inflammasomes: current understanding and open questions. Cell Mol Life Sci (2011) 68(5):765-83. doi:10.1007/s00018-010-0567-4

3. Kubes P, Mehal WZ. Sterile inflammation in the liver. Gastroenterology (2012) 143(5):1158-72. doi:10.1053/j.gastro.2012.09.008

4. Ekong UD, McKiernan P, Martinez M, Lobritto S, Kelly D, Ng VL, et al. Long-term outcomes of de novo autoimmune hepatitis in pediatric liver transplant recipients. Pediatr Transplant (2017) 21(6):1-8. doi:10.1111/petr.12945

5. Hennes EM, Zeniya M, Czaja AJ, Pares A, Dalekos GN, Krawitt EL, et al. Simplified criteria for the diagnosis of autoimmune hepatitis. Hepatology (2008) 48(1):169-76. doi:10.1002/hep.22322

6. Mieli-Vergani G, Heller S, Jara P, Vergani D, Chang MH, Fujisawa T, et al. Autoimmune hepatitis. J Pediatr Gastroenterol Nutr (2009) 49(2):158-64. doi:10.1097/MPG.0b013e3181a1c265

7. Chen KE, Richards AA, Caradoc-Davies TT, Vajjhala PR, Robin G, Lua LH, et al. The structure of the caspase recruitment domain of BinCARD reveals
[Human Investigation Committee]. All subjects gave written informed consent in accordance with the Declaration of Helsinki.

\section{AUTHOR CONTRIBUTIONS}

AA: concept design, performed experiments, data analysis, and writing first draft of manuscript; JY and AL: performed experiments, data analysis, and manuscript review; YA, MM, and SL: subject recruitment, sample collection, review of inclusion/exclusion criteria, and manuscript review; YD and GG: statistics, data analysis, and manuscript review; SM and JK: single-cell sequencing data analysis, manuscript writing, and manuscript review; GW: single cell sequencing, single cell sequencing data analysis, manuscript writing, and manuscript review; UE: concept design, data analysis, revising first and subsequent drafts of manuscript, subject recruitment, sample collection, and review of inclusion/ exclusion criteria.

\section{ACKNOWLEDGMENTS}

The authors would like to thank Drs. David Hafler, Fred Gorelick, and James Boyer for their critical review of our manuscript.

\section{FUNDING}

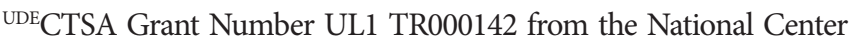
for Advancing Translational Science (NCATS), a component of the National Institutes of Health. UDE National Institute of Diabetes and Digestive and Kidney Diseases of the National Institutes of Health under Award Number P30KD034989.

\section{SUPPLEMENTARY MATERIAL}

The Supplementary Material for this article can be found online at https://www.frontiersin.org/articles/10.3389/fimmu.2018.01612/ full\#supplementary-material.

that all three cysteines can be oxidized. Acta Crystallogr D Biol Crystallogr (2013) 69(Pt 5):774-84. doi:10.1107/S0907444913001558

8. Wen H, Miao EA, Ting JP. Mechanisms of NOD-like receptor-associated inflammasome activation. Immunity (2013) 39(3):432-41. doi:10.1016/j. immuni.2013.08.037

9. Vande Walle L, Van Opdenbosch N, Jacques P, Fossos A, Verheugen E, Vogel P, et al. Negative regulation of the NLRP3 inflammasome by A20 protects against arthritis. Nature (2014) 512(7512):69-73. doi:10.1038/nature13322

10. Satija R, Farrell JA, Gennert D, Schier AF, Regev A. Spatial reconstruction of single-cell gene expression data. Nat Biotechnol (2015) 33(5):495-502. doi:10.1038/nbt.3192

11. Dominguez-Villar M, Baecher-Allan CM, Hafler DA. Identification of $\mathrm{T}$ helper type 1-like, Foxp3+ regulatory T cells in human autoimmune disease. Nat Med (2011) 17(6):673-5. doi:10.1038/nm.2389

12. Kitz A, de Marcken M, Gautron AS, Mitrovic M, Hafler DA, DominguezVillar M. AKT isoforms modulate Th1-like Treg generation and function in human autoimmune disease. EMBO Rep (2016) 17(8):1169-83. doi:10.15252/ embr.201541905

13. Dieude M, West LJ, Muruve DA, Gunaratman L, Mohanakumar T, Zorn E, et al. New answers to old conundrums: what antibodies, exosomes and inflammasomes bring to the conversation. Canadian National Transplant Research Program international summit report. Transplantation (2018) 102(2):209-14. doi:10.1097/TP.0000000000001872 
14. Westerterp M, Gautier EL, Ganda A, Molusky MM, Wang W, Fotakis P, et al. Cholesterol accumulation in dendritic cells links the inflammasome to acquired immunity. Cell Metab(2017) 25(6):1294-304.e6. doi:10.1016/j.cmet.2017.04.005

15. Yang H, Hreggvidsdottir HS, Palmblad K, Wang H, Ochani M, Li J, et al. A critical cysteine is required for HMGB1 binding to toll-like receptor 4 and activation of macrophage cytokine release. Proc Natl Acad Sci U S A (2010) 107(26):11942-7. doi:10.1073/pnas.1003893107

16. Kummer JA, Broekhuizen R, Everett H, Agostini L, Kuijk L, Martinon F, et al. Inflammasome components NALP 1 and 3 show distinct but separate expression profiles in human tissues suggesting a site-specific role in the inflammatory response. J Histochem Cytochem (2007) 55(5):443-52. doi:10.1369/ jhc.6A7101.2006

17. Wang CQ, Cruz-Inigo AE, Fuentes-Duculan J, Moussai D, Gulati N, SullivanWhalen M, et al. Th17 cells and activated dendritic cells are increased in vitiligo lesions. PLoS One (2011) 6(4):e18907. doi:10.1371/journal.pone.0018907

18. Zhang W, Cai Y, Xu W, Yin Z, Gao X, Xiong S. AIM2 facilitates the apoptotic DNAinduced systemic lupus erythematosus via arbitrating macrophage functional maturation. J Clin Immunol (2013) 33(5):925-37. doi:10.1007/s10875-013-9881-6

19. Yang CA, Huang ST, Chiang BL. Sex-dependent differential activation of NLRP3 and AIM2 inflammasomes in SLE macrophages. Rheumatology (Oxford) (2015) 54(2):324-31. doi:10.1093/rheumatology/keu318

20. Inoue M, Shinohara ML. NLRP3 inflammasome and MS/EAE. Autoimmune Dis (2013) 2013:859145. doi:10.1155/2013/859145

21. Inoue M, Williams KL, Gunn MD, Shinohara ML. NLRP3 inflammasome induces chemotactic immune cell migration to the CNS in experimental autoimmune encephalomyelitis. Proc Natl Acad Sci U S A (2012) 109(26):10480-5. doi:10.1073/pnas.1201836109

22. Kahlenberg JM, Thacker SG, Berthier CC, Cohen CD, Kretzler M, Kaplan MJ Inflammasome activation of IL-18 results in endothelial progenitor cell dysfunction in systemic lupus erythematosus. J Immunol (2011) 187(11):6143-56. doi:10.4049/jimmunol.1101284

23. Karni A, Koldzic DN, Bharanidharan P, Khoury SJ, Weiner HL. IL-18 is linked to raised IFN-gamma in multiple sclerosis and is induced by activated
CD4(+) T cells via CD40-CD40 ligand interactions. J Neuroimmunol (2002) 125(1-2):134-40. doi:10.1016/S0165-5728(02)00018-8

24. Volin MV, Koch AE. Interleukin-18: a mediator of inflammation and angiogenesis in rheumatoid arthritis. J Interferon Cytokine Res (2011) 31(10):745-51. doi:10.1089/jir.2011.0050

25. Nishio A, Tatsumi T, Nawa T, Suda T, Yoshioka T, Onishi Y, et al. CD14(+) monocyte-derived galectin-9 induces natural killer cell cytotoxicity in chronic hepatitis C. Hepatology (2017) 65(1):18-31. doi:10.1002/hep. 28847

26. Liaskou E, Zimmermann HW, Li KK, Oo YH, Suresh S, Stamataki Z, et al. Monocyte subsets in human liver disease show distinct phenotypic and functional characteristics. Hepatology (2013) 57(1):385-98. doi:10.1002/ hep. 26016

27. Tacke F, Randolph GJ. Migratory fate and differentiation of blood monocyte subsets. Immunobiology (2006) 211(6-8):609-18. doi:10.1016/j.imbio.2006. 05.025

28. Demetris AJ, Bellamy C, Hubscher SG, O’Leary J, Randhawa PS, Feng S, et al. Comprehensive update of the Banff Working Group on liver allograft pathology: introduction of antibody-mediated rejection. Am J Transplant (2016) 16(10):2816-35. doi:10.1111/ajt.13909

Conflict of Interest Statement: The authors declare that the research was conducted in the absence of any commercial or financial relationships that could be construed as a potential conflict of interest.

Copyright (C) 2018 Arterbery, Yao, Ling, Avitzur, Martinez, Lobritto, Deng, Geliang, Mehta, Wang, Knight and Ekong. This is an open-access article distributed under the terms of the Creative Commons Attribution License (CC BY). The use, distribution or reproduction in other forums is permitted, provided the original author(s) and the copyright owner(s) are credited and that the original publication in this journal is cited, in accordance with accepted academic practice. No use, distribution or reproduction is permitted which does not comply with these terms. 